\title{
Clinical analysis of 76 patients pathologically diagnosed with pulmonary cryptococcosis
}

\author{
Yuan Zhang*,§, Nan Li*,+, Yuxuan Zhang*„§, Huiping Li*, Xueyuan Chen*, \\ Shanmei Wang*, Xia Zhang*, Rongxuan Zhang ${ }^{\#}$, Jinfu Xu*, Jingyun Shi ${ }^{\star}$ \\ and Rex C. Yung ${ }^{+}$
}

ABSTRACT: The aim of the present study was to investigate the clinical characteristics of pulmonary cryptococcosis patients in China, with analysis of immunocompetent and immunocompromised subjects.

We performed a retrospective review of 76 patients diagnosed with tissue-confirmed pulmonary cryptococcosis at the Shanghai Pulmonary Hospital (Shanghai, China) during a 10-yr period (2001-2010).

Of 76 patients (54 males and 22 females), 41 (53.95\%) were immunocompetent and 35 out of the 41 were asymptomatic. Approximately $80 \%$ of the patients had histories suspicious of environmental fungal exposure. Radiological (computed tomography) findings showed predominantly peripheral findings (85.53\%, 65 out of 76 patients) including nodular masses $(55.26 \%$, 42 out of 76$)$, pneumonic infiltrates $(23.68 \%, 18$ out of 76$)$ and mixed type $(21.05 \%, 16$ out of 76$) .43 .42 \%$ (33 out of 76) were initially misdiagnosed, often as cancer by false-positive ${ }^{18} \mathrm{~F}$-fluorodeoxyglucose positron emission tomography $\left({ }^{18} \mathrm{FDG}-\mathrm{PET}\right)$ ( 28 out of 46 cases). 51 patients received antifungal therapy, 25 patients were clinically observed without treatment. As of December 31, 2010, 71 cases showed total recovery and four cases showed improvement (efficacy rate of $98.68 \%, 75$ out of 76 ). One HIVpositive case died of cryptococcal meningitis.

Incidence of pulmonary cryptococcosis in China may be related to environmental fungal exposures. Most presented as asymptomatic peripheral lung lesions. False-positive ${ }^{18}$ FDG-PET examinations often lead to initial clinical misdiagnosis of cancer. Unlike immunocompromised or clinically symptomatic patients, all immunocompetent patients had a good response, either to fluconazole monotherapy or observation, with a tendency for spontaneous remissions in the asymptomatic immunocompetent subjects.

KEYWORDS: Environmental exposure, immunocompetent host, pathological diagnosis, pulmonary cryptococcosis, serum cryptococcal antigen test

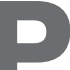

ulmonary cryptococcosis is an opportunistic infection. Most cases are caused by Cryptococcus neoformans or Cryptococcus gattii infection $[1,2]$. Previously, pulmonary cryptococcosis was thought to occur only in patients with AIDS and other immunocompromised states, such as patients undergoing organ transplantation or those on immunosuppressive regimens [3, 4]. Although immunocompromised hosts are considered to be at high risk for the life-threatening complications of cryptococcal pulmonary infection, pulmonary cryptococcosis can also occur in immunocompetent subjects $[5,6]$. Compared with $C$. neoformans, $C$. gattii is more inclined to cause disease in healthy people [7]. C. gattii infections have drawn increased attention since 2002 [8], with cases reported in Papua New Guinea and Northern Australia, India, Brazil, Vancouver Island in Canada, and Washington State and Oregon in the USA. However, C. gattii has been rare in China: FENG et al. [9] reported only nine out of 110 clinical Cryptococcus isolates (8.2\%) from China as C. gattii, with the vast majority were $C$. neoformans var. grubii, which is in accord with the data of most studies of clinical molecular epidemiology in other geographic areas [10, 11].

Pulmonary cryptococcosis is not rare on the Chinese mainland [12-14]. Our present understanding of pulmonary cryptococcosis is mostly
AFFILIATIONS

*Dept of Respiratory Medicine, Shanghai Pulmonary Hospital, Tongji University School of Medicine, "Dept of Pathology, Shanghai Pulmonary Hospital, Tongji University School of Medicine, and "Dept of Radiology, Shanghai Pulmonary Hospital, Tongji University School of Medicine, Shanghai, China.

${ }^{+}$Dept of Pulmonary and Critical Care Medicine, Johns Hopkins University School of Medicine, Baltimore, MD, USA.

${ }^{\S}$ These authors contributed equally to this work.

CORRESPONDENCE

H. Li

Dept of Respiratory Medicine, Shanghai Pulmonary Hospital Tongji University School of Medicine 507 Zhengmin Road Shanghai 200433 China E-mail: liw2013@126.com

Received:

Sept 282011

Accepted after revision: Feb 162012 First published online: March 092012 
limited to typical clinical manifestations and radiological presentations. In fact, the great variations and protean manifestations of these clinical features also often lead to misdiagnosis. In addition, the treatment protocol for pulmonary cryptococcosis, especially for immunocompetent patients, is not clearly stated. Here, we performed a retrospective evaluation of 76 patients with a tissue-confirmed diagnosis of pulmonary cryptococcosis over a period of 10 yrs (2001-2010) at our hospital. Our specific aims were to provide an updated review and to characterise the epidemiological, clinical, immunological, radiological characteristics, as well as treatment of pulmonary cryptococcosis in China.

\section{MATERIALS AND METHODS \\ Patients}

A retrospective review based on discharge summaries from January 2001 to December 2009 was performed; 76 consecutive patients with tissue-proven pulmonary cryptococcosis verified by biopsy at Shanghai Pulmonary Hospital (Shanghai, China) were identified and included in this review. Patients who were diagnosed with pulmonary cryptococcosis on the basis of cryptococcal antigen plus radiographic (computed tomography (CT) and positron emission tomography (PET)) findings that are consistent with pulmonary cryptococcosis, but without histological confirmation, were excluded. The medical records of all patients, including demographics, underlying diseases, respiratory symptoms, laboratory tests including immune status studies, imaging data, treatment and outcome were collected. The relevant follow-up patient information was obtained on regular clinic visits and by telephone follow-up. The last followup was on December 31, 2010. The study plan has been approved by Shanghai Pulmonary Hospital's Ethics Committee (2011-FK112), and the patients' informed consents for participating of the study were obtained prior to data collection or analysis.

\section{Pathological diagnosis}

\section{Histology}

Diagnosis of pulmonary cryptococcosis was confirmed in 76 patients with tissue biopsy for pathological findings. Of the 76 cases, 68 cases received either a thoracoscopic surgery or openlung biopsy, seven cases underwent transthoracic aspiration and one case was diagnosed bronchoscopically by transbronchial lung biopsy (TBLB). All samples were fixed using conventional $4 \%$ neutral formalin, embedded in paraffin, stained with haematoxylin-eosin and histochemically stained with periodic acid-Schiff (PAS), mucus card Red (Mc) and Grocott's methenamine silver (GMS). The samples were then examined by light microscopy [14]. Thus, the presence of the cryptococcal pathogen in the tissues sections was the direct histological evidence of pulmonary cryptococcosis.

\section{Culture}

The specimens (serum, blood, bronchoalveolar lavage fluid (BALF), tissue, sputum from patients or air samples from patients' living environments) were cultured on glucose agar culture medium at room temperature $\left(25^{\circ} \mathrm{C}\right)$ or $37^{\circ} \mathrm{C}$ for $2-$ 5 days for fungi.

\section{Radiological assessment}

All pulmonary cryptococcosis patients during this period had a CT scan. The CT scans were carried out with one of two multislice detection scanners (Brilliance 40 or Brilliance 64;
Philips, Amsterdam, the Netherlands) at the Shanghai Pulmonary Hospital. Consecutive 8-mm thick sections were obtained from the lung apices to the lung bases with additional 1-2-mm thin sections through areas with abnormal lesions. The main manifestations of CT findings were mainly classified into three patterns, namely nodular, pneumonia and mixed morphological characteristics. 1) The nodular imaging finding with a clear boundary was further subdivided divided into single nodular (fig. 1a and b) and multinodular (fig. 1c). 2) The pneumonic infiltrates with ill-defined margins were subcategorised as single and multifocal pneumonia (fig. $1 \mathrm{~d}$ and e). 3) In mixed morphological patterns, both nodular and patchy infiltrates were detected (fig. 1f). Cavity, pleural effusion, mediastinal lymphadenopathy and air bronchograms were also recorded accordingly. Two thoracic radiologists who were unaware of the patients' immune statuses and clinical symptoms assessed all findings independently.

The brain CT and magnetic resonance imaging, when performed, were recorded to evaluate who may have central nervous system (CNS) involvement.

\section{Immune function tests and evaluation}

The following tests for humoral or cellular immunity impairments were measured to determine status of immune function and impairment.

1) Humoral immune parameters, including serum immunoglobulin (Ig)G, IgA and IgM were quantitatively measured using immunoturbidimetry. Patients were classified as immune impaired when any two values were below the lower limits of the normal range $\left(\operatorname{IgG} 7-16 \mathrm{~g} \cdot \mathrm{L}^{-1}, \operatorname{IgA} 0.4-2.3 \mathrm{~g} \cdot \mathrm{L}^{-1}\right.$ and $\operatorname{IgM}$ 0.7-4.0 $\left.\mathrm{g} \cdot \mathrm{L}^{-1}\right)$.

2) Cellular immune parameters, including peripheral blood count and differential (the total count and classification of white blood cells), and serum CD3, CD4 and CD8 were measured using flow cytometry. The cellular immune functions were regarded as normal when the percentage of CD3 cells ranged from $62 \%$ to $76 \%$, the percentage of CD4 cells ranged from $30 \%$ to $40 \%$ and the CD4: CD8 ratio ranged from 1.5 to 2.0 .

3) Patients with a past history of at least one of the predisposing conditions, including use of immunosuppressive drugs (treatment with corticosteroids or disease-modifying drugs with immunosuppressive effects), severe diabetes mellitus associated with organ damage, malignancies, HIV infection, history of organ transplantation, severe respiratory system limitation or other systemic disorders (e.g. collagen vascular disease such as lupus) and being on steroids, were considered immunocompromised. Patients were also considered immunodeficient if their peripheral absolute neutrophil count was $<2.0 \times 10^{4}$ cells $\mu \mathrm{L}^{-1}$ or absolute lymphocyte count was $<1,000$ cells $\cdot \mu \mathrm{L}^{-1}$.

\section{Serum cryptococcal antigen test}

The result of a serological latex agglutination test was considered positive when the titre $\geqslant 1: 8$.

\section{Clinical outcome evaluating criteria}

\section{Cured/complete response}

Cured/complete response was defined as clinical symptoms having resolved and imaging findings indicating that all lesions have completely disappeared. 

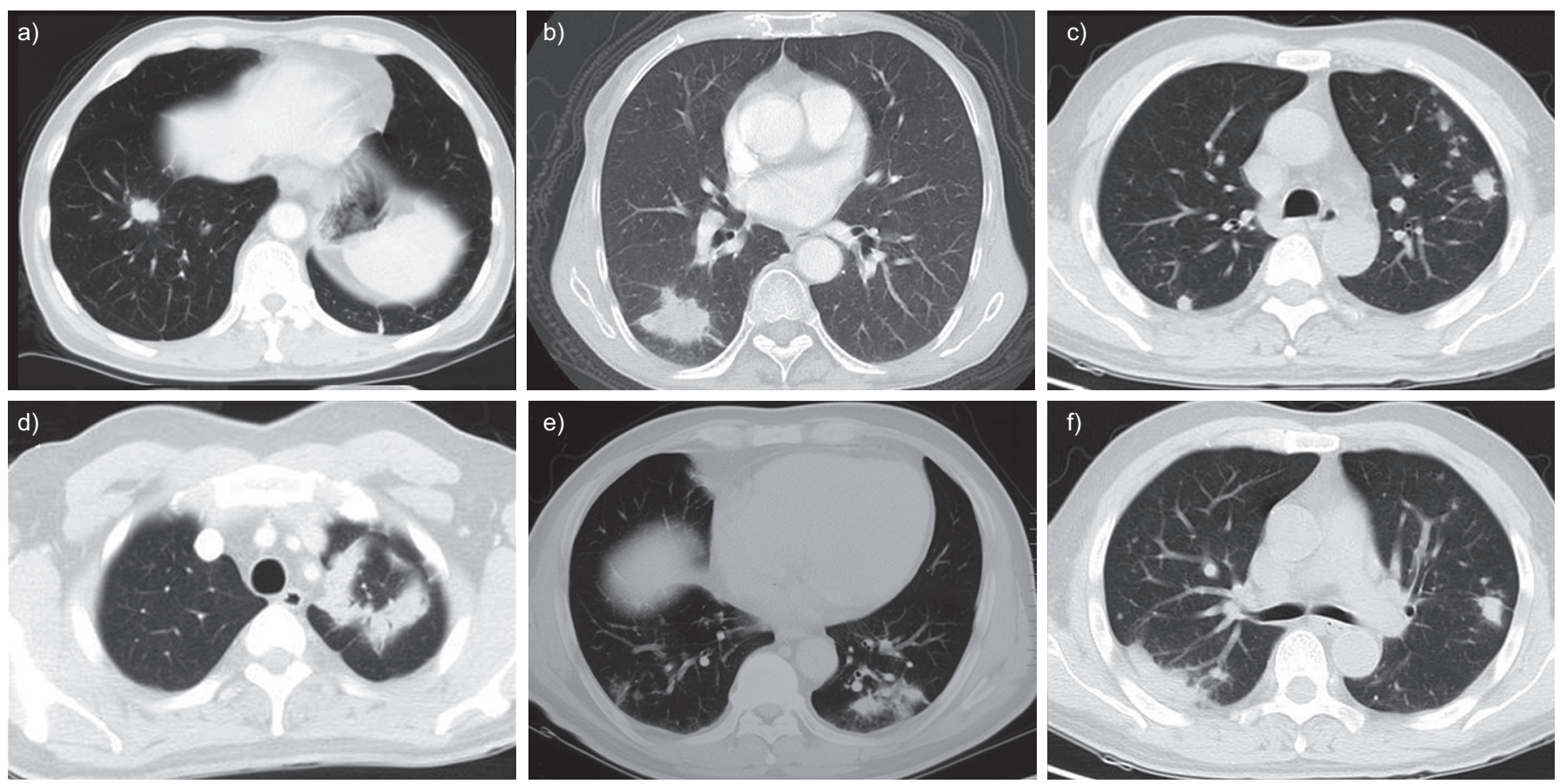

FIGURE 1. The findings of computed tomography scans vary: a) a single small nodule; b) a single large nodule; c) multiple lung nodules; d) pneumonia, with a single focus of a dense pulmonary infiltrate; e) multiple patches with lower density shadows; f) mixed, both nodule and pneumonic foci in bilateral lungs. Magnification: $40 \times$.

\section{Partial response}

Partial response was defined as clinical symptoms having improved and imaging findings indicating that the lesions had partially resolved.

\section{No change/progressive disease}

No change was defined as clinical symptoms and imaging findings having not changed or the clinical symptoms having deteriorated, Progressive disease was defined as imaging indicating that the lesions had progressed.

\section{Statistical analysis}

All the information was entered in an approved research database by three individuals independently, after verification, with the SPSS 16.0 statistical analysis software (IBM, Armonck, NY, USA) for data processing. All data are presented as mean \pm SD. The Chi-squared test was used for ordinal data, the unpaired t-test was used for numerical data and the nonparametric test for ranked data. Significance was set to $p<0.05$ for all statistical analysis.

\section{RESULTS}

\section{Demographic information}

Our retrospective analysis identified 264 patients with a hospital discharge of pulmonary cryptococcosis, these included 76 patients with tissue-proven PC. These 76 patients (54 males and 22 females) ranged from 19 to 72 yrs of age (median 50 yrs; mean \pm SD $49.76 \pm 10.87 \mathrm{yrs}) .21(27.63 \%)$ patients had a history of smoking.

\section{Immune function}

$41(53.95 \%)$ patients had no comorbidity or immune dysfunction; $35(46.05 \%)$ cases that had abnormal testing results of the humoral immune or cell immune dysfunction and were considered immunocompromised (table 1). These 35 patients had past history of at least one of the predisposing conditions: use of immunosuppressive drugs, severe diabetes mellitus with organ damage, malignancy, HIV infection, and respiratory system or other system serious disorders.

\section{Environmental exposures}

$\sim 80 \%$ of patients had a history of environmental exposure (a clear history of exposure to pigeon droppings, dust or fungal spores or keeping cats, dogs or poultry) (table 2).

\section{Clinical manifestations}

In this group, 45 (59.21\%) patients had a variety of symptoms, including cough (41 cases), productive of sputum (35 cases), chest pain (16 cases), fever (14 cases), shortness of breath (12 cases), chest tightness (12 cases), fatigue and discomfort (nine cases), sweating (seven cases), minimum blood streaking in the sputum (seven cases) and gross haemoptysis (four cases). All of the 35 immunocompromised patients had at least one or more of the above symptoms. Conversely, most of the immunocompetent pulmonary cryptococcosis patients (31 out of $41,82.93 \%$ ) did not present with any symptom and their pulmonary diseases were detected by incidental radiological examination (chest radiography or CT scan). One HIV-positive patient showed abnormal CNS imaging by head CT. Overall, symptoms are much more likely in immunocompromised patients (35 out of $35,100 \%$ ) than in immunocompetent patients (10 out of 41 , $24.39 \%)(\mathrm{p}<0.05)$.

\section{Serological latex agglutination test}

Of the 76 patients reviewed, seven had a serum cryptococcal antigen (sCRAG) test and five showed positive results (titre 1:320-1:1280). 
TABLE 1 The past medical history of 35 immunocompromised patients with pulmonary cryptococcosis

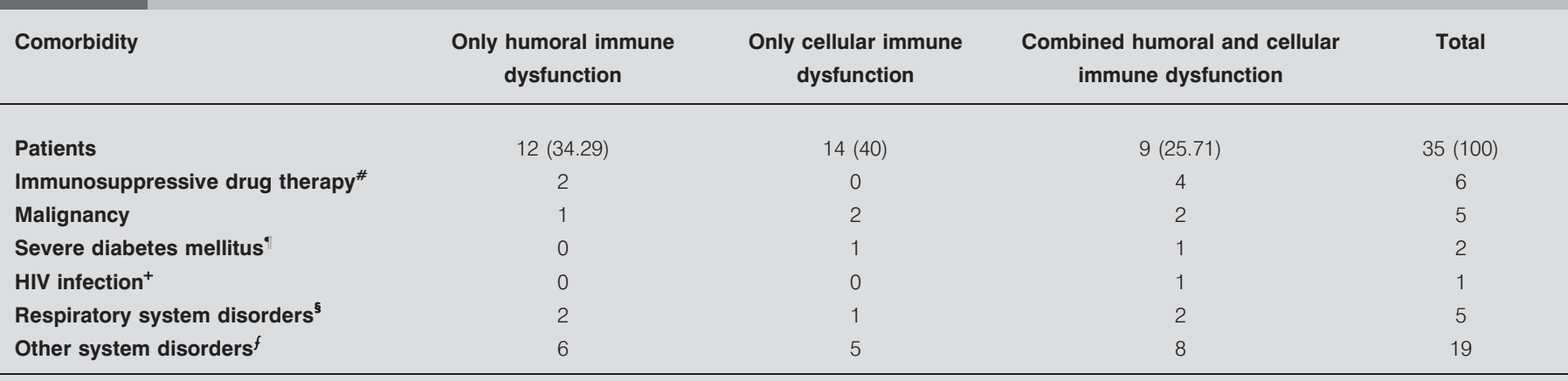

Data are presented as $\mathrm{n}(\%)$ or $\mathrm{n} .^{\#}$ : glucocorticoids, $>10 \mathrm{mg}$ for $>3$ months within the previous half year; " ${ }^{*}$ : diabetes mellitus with organ damage; ${ }^{+}$: CD4+ $\mathrm{T}^{- \text {cell }}$ count $<200$ cells $\cdot \mathrm{mm}^{-3}$ at time of pulmonary cryptococcosis diagnosis; ${ }^{5}$ : previous tuberculosis $(n=2)$, chronic bronchitis $(n=2)$ and bronchial asthma $(n=1) ;{ }^{f}:$ autoimmune diseases and collagen diseases $(n=4)$ (systemic lupus erythematosus $(n=1)$, dermatomyositis $(n=1)$ and scleroderma $(n=2))$, rheumatoid arthritis $(n=3)$, hepatitis $B$ $(n=2)$, nephrotic syndrome $(n=1)$ and cardiovascular diseases $(n=9)$ (coronary heart disease $(n=4)$, severe arrhythmia $(n=2)$ and hypertension $(n=3)$ ).

\section{Sputum examination}

A sputum examination was performed on all patients in this group. C. neoformans grew from only two cultures, for a culture positivity rate of $2.63 \%$.

\section{Findings on chest imaging $\mathrm{CT}$ scan}

Of the 76 patients, lung lesions of $85.53 \%$ (65 out of 76 ) patients were located mostly in the peripheral lung field (outer third of the lung), close to the pleura. Lesions in $39(51.32 \%)$ patients involved only the right lung, $22(28.95 \%)$ patients only the left lung and were bilateral in 15 (19.74\%) (table 3).

Lesions were varied in shape (fig. 1), including single or multiple nodules, pneumonic infiltrates or both (mixed) (table 4). 42 cases had nodular masses, 18 cases had pneumonic infiltrates and 16 cases were of a mixed type. $68.30 \%$ of immunocompetent patients (28 out of 41 ) showed either single or multi nodular lesions. $40 \%$ of immunocompromised patients displayed nodular lesions, the other $60 \%$ cases showed pneumonia or mixed morphology.

\section{${ }^{18} \mathrm{~F}$-fluorodeoxyglucose positron emission tomography}

46 patients in this group of 76 patients underwent ${ }^{18}$ FDG-PET. In total, $28(60.87 \%)$ cases displayed abnormal uptake standardised uptake values (SUV) $>2.5$ and a lesion-to-normal tissue ratio $(\mathrm{T}: \mathrm{N}) \geqslant 1.3$ in the lung lesions, which heightened the suspicion for lung cancer; four cases showed abnormal uptake with a mild increase in SUV suspicious for nonmalignant inflammation; and 14 cases showed normal ${ }^{18} \mathrm{FDG}$ uptake, indicating no active inflammation.

\section{Tissue acquisition}

Overall, 68 cases of pulmonary cryptococcosis were confirmed by surgical biopsy, seven by percutaneous needle lung biopsy and one by TBLB via fibre-optic bronchoscope. Post-operative pathology by PAS, Mc, six-ammonium silver staining or GMS revealed cryptococcal-infected granuloma (fig. 2) or a more mucus-like substance in association with cryptococcal pathogen.

\section{Diagnosis}

The time from clinical presentation to final tissue diagnosis ranged from 17 days to $1 \mathrm{yr}$ (mean \pm SD $65 \pm 52$ days; $67 \pm 45$ days for immunocompetent versus $64 \pm 60$ days for immunocompromised groups, $\mathrm{p}>0.05)$. Of all 76 cases, cryptococcal infection was considered in only four $(5.26 \%)$ cases on the initial visits. The diagnoses of the preliminary testing in $39(51.32 \%)$ cases were uncertain, although they were later confirmed as pulmonary cryptococcosis by biopsy. The misdiagnosis rate at presentation was $43.42 \%$ (33 out of 76); 23 cases were misdiagnosed as lung cancer $\left({ }^{18} \mathrm{FDG}\right.$-PET also suggested lung cancer in these cases), eight cases as lung bacterial infection and two cases as tuberculosis.

TABLE 2 History of environmental exposure of 76 patients with diagnosis of pulmonary cryptococcosis

\begin{tabular}{|c|c|c|c|}
\hline Occupational environment exposure history & Patients & Immunocompetent patients & Immunocompromised patients \\
\hline History of exposure to pigeon droppings & $26(34.21)$ & 12 & 14 \\
\hline History of dust or fungal spores & $19(25)$ & 9 & 10 \\
\hline History of keeping cats, dogs or poultry & $16(21.05)$ & 11 & 5 \\
\hline No exposure history & $15(19.74)$ & 10 & 5 \\
\hline Total & $76(100)$ & 41 & 35 \\
\hline
\end{tabular}

Data are presented as $\mathrm{n}(\%)$ or $\mathrm{n}$. 


\begin{tabular}{|c|c|c|c|c|}
\hline TABLE 3 & $\begin{array}{l}\text { esions dis } \\
\text { ulmonary }\end{array}$ & $\begin{array}{l}\text { ution in } 7 \\
\text { ptococco }\end{array}$ & atients w & \multirow{3}{*}{ Total } \\
\hline \multirow[t]{2}{*}{ Variables } & \multirow[t]{2}{*}{ Bilateral } & \multicolumn{2}{|c|}{ Unilateral } & \\
\hline & & Left & Right & \\
\hline Patients & 15 (19.74) & 22 (28.95) & $39(51.32)$ & $76(100)$ \\
\hline \multicolumn{5}{|l|}{ Lobes } \\
\hline Upper & $4(5.26)$ & $9(11.84)$ & $9(11.84)$ & 22 (28.95) \\
\hline Middle & NA & NA & $10(13.16)$ & $10(13.16)$ \\
\hline Lower & $6(7.89)$ & $11(14.47)$ & $16(21.05)$ & $33(43.42)$ \\
\hline Multilobar & $5(6.58)$ & 2 (2.63) & $4(5.26)$ & $11(14.47)$ \\
\hline \multicolumn{5}{|l|}{ Lung field $^{\#}$} \\
\hline $\begin{array}{l}\text { Near the } \\
\text { hilum }\end{array}$ & 2 & 1 & 1 & $4(5.26)$ \\
\hline Middle third & 1 & 2 & 4 & $7(9.21)$ \\
\hline Outer third & 12 & 19 & 34 & 65 (85.53) \\
\hline
\end{tabular}

Data are presented as n (\%) or n. NA: not applicable. ${ }^{*}$ : centre of lesion.

\section{Treatment and outcome}

As shown in table 5, 51 patients received antifungal treatments. The duration of the treatment ranged from 2 weeks to 2 yrs, with a mean of 4.8 months and a median of 3 months. Followups ended on December 31, 2010 (12-114 months, mean \pm SD $58.82 \pm 36.98$ months). In the 76 patients, 71 cases were cured and four cases showed improvement (overall efficiency $98.68 \%$ ). There was only one death, in the HIV-positive patient (CD4 count $<200$ cells $\cdot \mathrm{mm}^{-3}$ ), complicated by cryptococcal meningitis. This patient died of cryptococcal encephalitis on the fourth day after a video-assisted thoracoscopic lung biopsy.

\section{DISCUSSION}

In the present study of a single-institution, retrospective review of 76 patients pathologically diagnosed with pulmonary cryptococcosis, we found that pulmonary cryptococcosis is not rare in immunocompetent persons, whose disease is often an asymptomatic radiological presentation and whose clinical outcome is most often favourable. Recent data showed that the incidence of pulmonary cryptococcosis is increasing. An epidemiological demographic study in British Columbia, Canada [2] showed the annual incidence of pulmonary cryptococcosis has increased from six per million in 1999 to 38 per million in 2006. This greater than six-fold increase cannot be explained by the common association with poorly controlled HIV patients, as it was reported that the incidence of people with HIV infection is stable. The increased incidence is mainly due to non-HIV infection [2]. At present, there are no data in China on the incidence of pulmonary cryptococcosis based on comprehensive epidemiological survey. The review by ZHANG [12] summarised 113 reports of 728 cases of pulmonary cryptococcosis in China from 1981 to 2009 . The first pulmonary cryptococcosis case was reported by ZHU and ZHANG [15] in 1981, an additional 65 cases were reported from 1981 to 2001 and another 668 cases were reported from 2001 to 2009. While the number of pulmonary cryptococcosis cases being reported in China has increased significantly during recent decades, it is uncertain if this is a true increase in incidence or secondary to presumed infections based on wider serological testing and more detailed chest imaging with greater access to $\mathrm{CT}$. In the review by ZHANG [12], 102 reports included detailed medical history and a total of $404(69.7 \%)$ out of 580 cases had no comorbidities that could explain an underlying immunosuppression. Our present cohort of 76 tissue-proven pulmonary cryptococcosis patients is the largest single group of patients with pulmonary cryptococcosis diagnosed by pathological examination in China. Notably, similar to the series of ZHANG [12], immunocompetent patients accounted for more than half $(53.95 \%)$ of the patients in the series. Even so, the single HIV patient in our cohort may seem inordinately low; however, medical care is organised in China in such a way that known HIV-positive patients are preferentially referred to infectious diseases hospitals and centres, whereas our institution is specialised in pulmonary-focused diseases.

With regard to sex distribution of pulmonary cryptococcosis, our cohort of 76 patients included 54 males and 22 females.

\section{TABLE 4 The relationship between imaging lesion patterns and immune function}

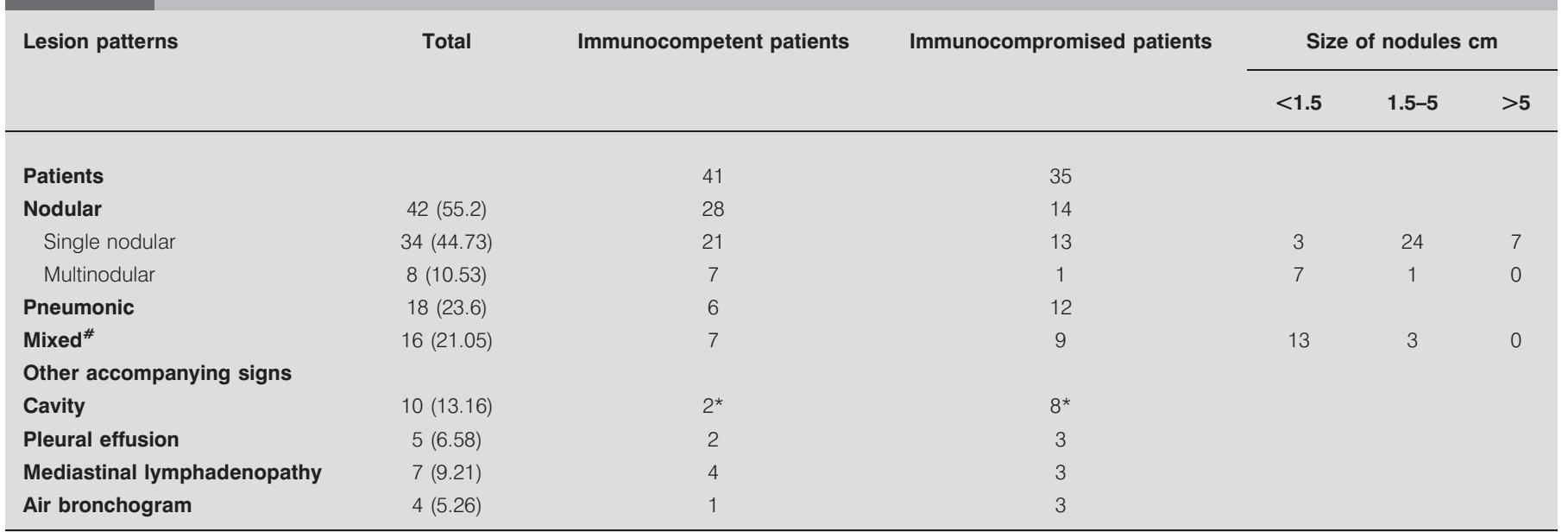

Data are presented as $n(\%)$ or $n$, unless otherwise stated. ${ }^{*}$ : nodular and pneumonic; *: $p<0.05$ between groups 

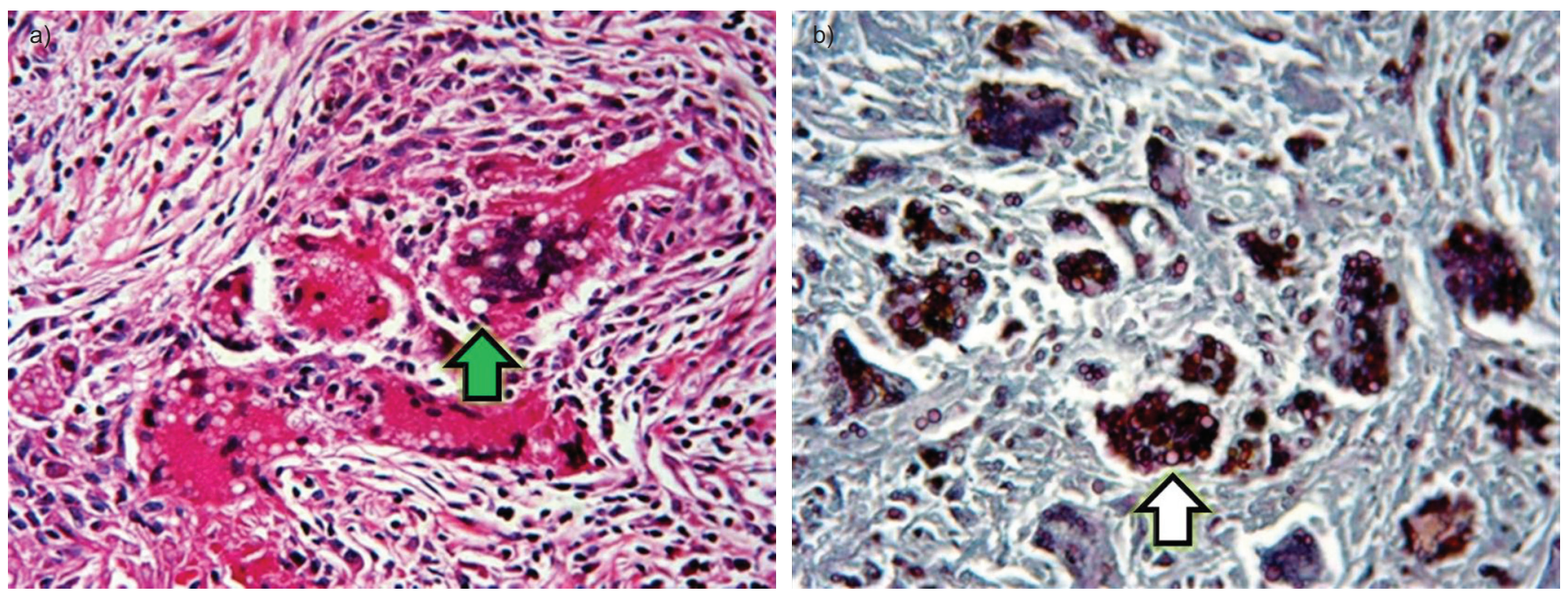

FIGURE 2. Lung biopsy. a) Inflammatory granuloma can be observed by haematoxylin-eosin staining; round cryptococcal spores can be seen (green arrow). b) Round cryptococcal spores can be best seen using Grocott's methenamine silver staining (white arrow)

The male:female ratio $(2.5: 1)$ in this group is similar to a previous pulmonary cryptococcosis report's ratio in China (2.3:1, 487 males and 216 females) [12].

Besides host immune factors, pulmonary cryptococcosis could also be related to environmental exposure to contaminated airspace, including close contact with animals [16-19], green plants [20] or other natural sources contaminated by fungi [21]. Cryptococcus is an encapsulated yeast that can be found in the faeces of birds, especially pigeons [16, 17]. Although there have been no reports of direct animal-to-human transmission, animals can carry cryptococcal spores and may contaminate the surrounding environments including trees, water and soil through bird droppings. Their ubiquitous presence in the environment could also become an important source of cryptococcal infections, as flying birds can increase fungal spore counts in the air [22]. In addition, household pets such as cats [18] can carry pathogenic Cryptococcus. As figure 3 shows,

\begin{tabular}{|c|c|c|c|c|c|}
\hline TABLE 5 & \multicolumn{5}{|c|}{$\begin{array}{l}\text { Treatment and outcome of } 76 \text { patients with } \\
\text { pulmonary cryptococcosis }\end{array}$} \\
\hline \multirow{2}{*}{\multicolumn{2}{|c|}{ Treatment methods }} & \multirow[t]{2}{*}{ Cases } & \multicolumn{3}{|c|}{ Outcomes } \\
\hline & & & CR & PR & NC/PD \\
\hline \multicolumn{6}{|c|}{ Antifungal treatment } \\
\hline Fluconazo & & 39 (51.32) & 39 & 0 & 0 \\
\hline Itraconazo & & $7(9.21)$ & 7 & 0 & 0 \\
\hline Amphoteri & B + 5-fluorocytosine & $5(6.58)$ & 1 & 3 & $1^{\#}$ \\
\hline $\begin{array}{l}\text { Clinical obs } \\
\text { therapy }\end{array}$ & rvation without & 25 (32.89) & 24 & 1 & 0 \\
\hline Total & & $76(100)$ & 71 & 4 & $1^{\#}$ \\
\hline
\end{tabular}

Data are presented as $n(\%)$ or $n$. CR: cured/complete response; PR: partial response; NC/PD: no change/progressive disease. ": this patient died of cryptococcal encephalitis on the fourth day after video-assisted thoracoscopic lung biopsy. the cryptococcal spores can spread freely in the air and eventually infect humans by inhalation.

Fungal contamination can occur not only in the living area (fig. 4) or office environment but also in automobiles, trains and other modern means of transportation. In particular, heating, ventilation and air-conditioning systems tend to foster fungal and bacterial contamination $[23,24]$. It has been reported that dust in the home environment also carries the potential for Cryptococcus contamination and subsequent exposure risk as high as $13 \%$ [21].

Of those surveyed in this study, $34.21 \%$ of patients (26 out of 76 ) had a history of direct or indirect exposure to pigeon droppings (e.g. from breeders who feed pigeons and sell pigeons at markets, veterinarians and bird fanciers feeding pigeons in the town square), which is much higher than the $12.8 \%$ from a

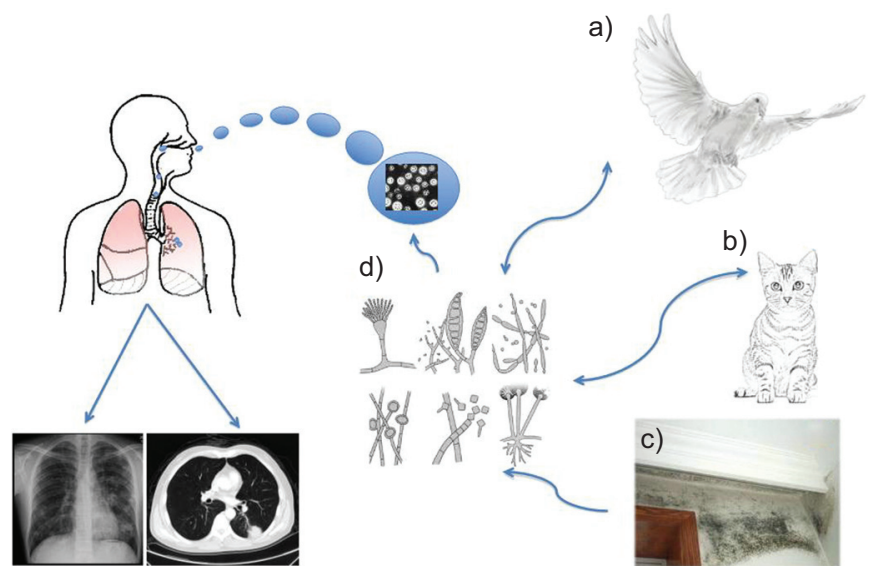

FIGURE 3. Schematic of potential sources of primary cryptococcal infection. The fungal spores can spread freely in the air and eventually infect humans through inhalation. a) Pigeons play a very important role in the course of disease transmission; b) pets such as cats can also carry fungus and spread them by direct contact; c) fungal contamination can occur in the living area; d) plants also can be an important source of fungus. 

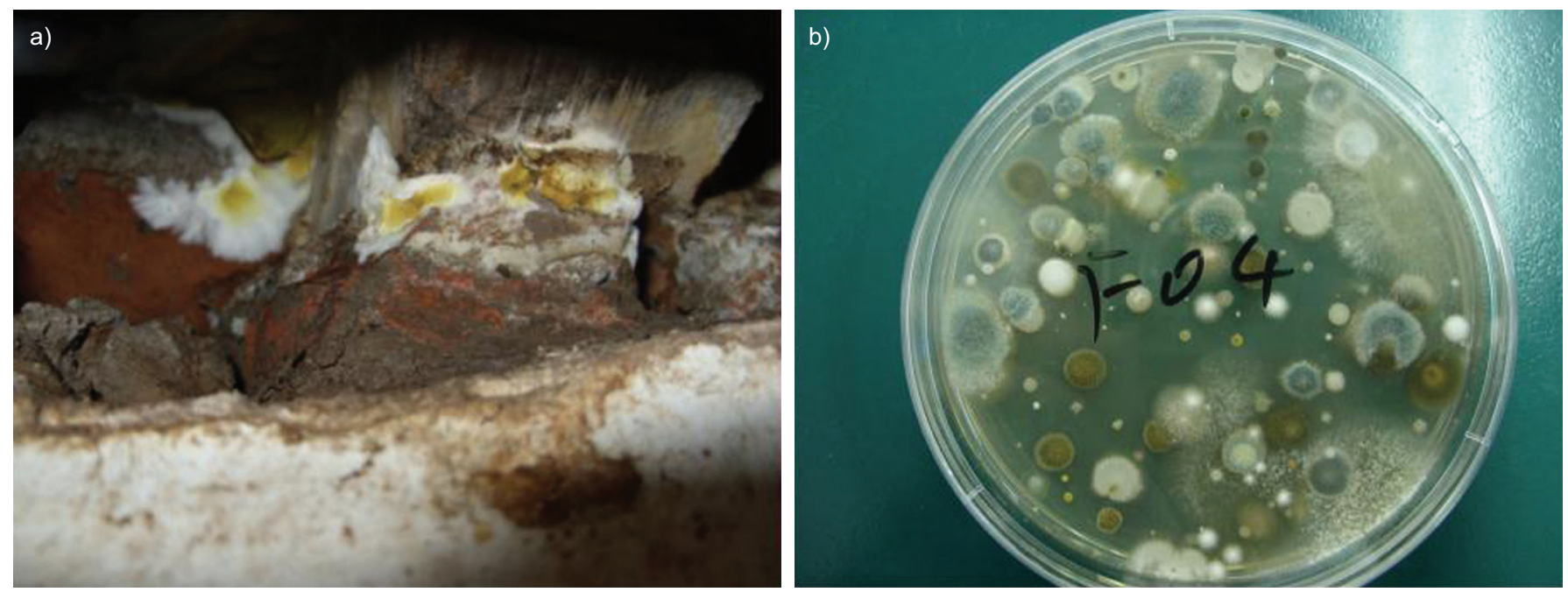

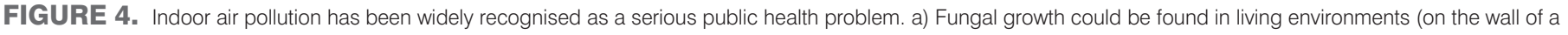
patient's bedroom); b) analysis of the ambient room air, microbiological plating demonstrating fungus growth.

previous report [12]. For $\sim 25 \%$ of our patients in the present series, their working or living environments also were potentially contaminated by fungal spores; two case examples are illustrated in figures $3 \mathrm{c}$ and 4 . This number is again higher than the $13.5 \%$ previously reported in China [12]. Although in this study, the environmental exposure was not significantly different $(\mathrm{p}=0.11)$ for immunocompromised and the immunocompetent patients, a large number $(81.26 \%)$ of pulmonary cryptococcosis patients had environmental exposure history, suggesting that clinicians should ask detailed questions about occupational and environmental exposure history.

In this group, most immunocompetent pulmonary cryptococcosis patients ( 31 out of $41,82.93 \%$ ) were without any pulmonary symptoms to suggest a lung infection and their pulmonary cryptococcosis was only detected by incidental imaging findings ordered as a part of routine annual examination or for other nonpulmonary indications.

The radiological presentations varied; however, the lesion patterns had some relevance to host immune function $(\mathrm{p}<0.05)$. $68.30 \%$ (28 out of 41 ) of immunocompetent patients showed nodular mass. Pneumonic and mixed types were more common in immunocompromised patients (12 plus nine out of 35,60\%) versus immunocompetent patients (six plus seven out of 41, $31.71 \%)(\mathrm{p}<0.05)$. Cavitation combined with pulmonary infiltrates was again more common in immunocompromised cases (eight out of 35 versus two out of $41, \mathrm{p}<0.05$.).

Although tissue biopsy and or a positive fungal culture for cryptococcal organisms are the most accurate means of confirming a definitive pulmonary cryptococcosis diagnosis, there are other noninvasive diagnostic tests, such as the sCRAG test. sCRAG testing is considered an effective noninvasive diagnostic tool, and is less complex and costly than the invasive procedures [25]. However, sCRAG testing has not been widely available in China; for example, during the period under study, only three hospitals in metropolitan Shanghai offered this test, and the cost of 400 renminbi per test is often prohibitively expensive for the average self-paying patient. This explains one of the shortcomings of our study, that only seven patients in our group underwent a sCRAG test and five cases yielded positive results (titre $>1: 320$ ) at a sensitivity of $71.4 \%$. Another limitation to our study is that few samples from either BALF or tissue obtained by biopsies were sent for culture. Most of the surgical excisions were directed towards resection of a suspected lung cancer, and this is the main reason that pulmonary cryptococcosis or other granulomatous infection had not been considered prior to the final pathological diagnosis.

Here, a major question is how one should choose among the different biopsy approaches appropriately. Different methods have respective advantages and disadvantages. Surgical morbidities are serious risks, and the costs are high, especially when applied to nonmalignant conditions. Both TBLB and percutaneous lung biopsy are less invasive, economical and practical, although smaller tissue samples are obtained. Our data suggest that $85.53 \%$ ( 65 out of 76 ) lesions of pulmonary cryptococcosis are located in the peripheral regions of the lungs. Percutaneous lung biopsy operation in experienced hands is relatively simple, less invasive and effective. With a definitive diagnosis rate of $74 \%$ to $95 \%$ [4-6], percutaneous lung biopsy may be the preferred initial biopsy method.

The initial clinical misdiagnosis rate of patients in this group was quite high, $43.42 \%$ (33 out of 76), patients being misdiagnosed with lung cancer (23 cases, 30.26\%), pneumonia (eight cases, $10.53 \%$ ) or tuberculosis (two cases, $2.63 \%$ ). Of special interest is that an ${ }^{18}$ FDG-PET examination was obtained in 46 out of 76 patients in this cohort, based on a clinical suspicion of cancer. SUV is used as a quantitative indicator of the ${ }^{18} \mathrm{FDG}$ tracer that is absorbed by tissue, and is usually used to distinguish malignant and benign lesions. For example, cancer is suggested when ${ }^{18}$ FDG-PET SUV in lung nodules is $>2.5$. Studies have reported a $94 \%$ accuracy of the use of this one criteria for lung cancer [26] However, ${ }^{18}$ FDG is a nonspecific tumour-imaging agent, and in addition to tumour tissues, nonmalignant tissue outside the tumour and some benign but inflammatory or infectious lesions can also demonstrate increased tissue uptake of ${ }^{18} \mathrm{FDG}$ [27]. It has been suggested that ${ }^{18}$ FDG-PET had a fairly low specificity of 

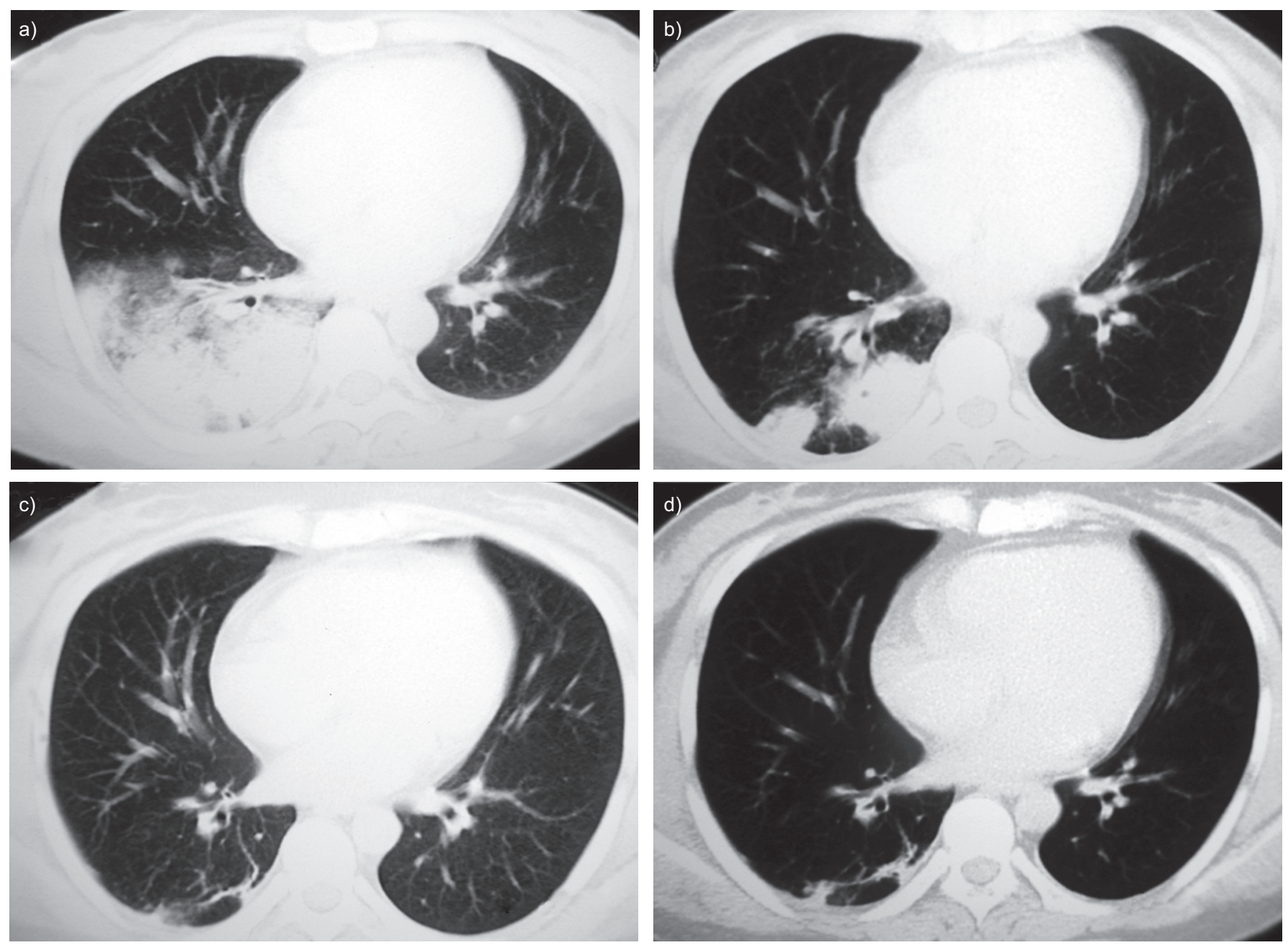

FIGURE 5. Chest imaging of a 34-yr-old, female, immunocompromised patient before and after treatment. a) Pre-treatment computed tomography (CT) scan. b) Followup CT scan after treatment with fluconazole monotherapy for 35 days. c) Follow-up CT scan after fluconazole treatment for 12 months. d) Follow-up CT scan after medication cessation for 4 months (total 16 months).

$58.97 \%$ but good sensitivity of $87.5 \%$ in a multicentre clinical trial on the diagnostic value of dual-tracer FDG-PET in pulmonary lesions $(n=55)$ [28]. In $60.87 \%$ (28 out of 46$)$ of the patients in our cohort, a high degree of abnormal uptake in the lung lesions (SUV > 2.5, T/N >1.3), which typically indicates malignancies resulted in rushing the patient directly to the operating table. Therefore, although ${ }^{18}$ FDG-PET has a definite role in directing the diagnosis and staging of thoracic malignancies, false-positive misdiagnosis of pulmonary cryptococcosis and other granulomatous diseases must be considered, especially in areas endemic for these infections.

In recent decades, the treatment of pulmonary cryptococcosis in China follows the cryptococcal treatment guidelines established by the Infectious Diseases Society of America, initially published in 2000 [29] and updated in 2010 [1]. These guidelines recommend that patients with pulmonary cryptococcosis should first be evaluated in accordance with the patient's immune status and for the extent of systemic spread. Patients should receive step-wise graded treatment according to the severity of respiratory symptoms and the presence of extrapulmonary spread and manifestation of disease. Accordingly, the immunocompromised patients $(n=35)$ in this present cohort were given a sufficient course of antifungal treatment. Of the overall 76 cases, 39 patients with mild-to-moderate symptoms were prescribed fluconazole monotherapy and had good response, a typical case of which is presented in figure 5 . There is controversy regarding the need for therapy in asymptomatic patients with normal immune function. In the immunocompetent patients in this series $(n=41), 25$ asymptomatic cases did not receive any antifungal therapies but were observed. Among them, 24 patients had complete resolution of radiographic findings and one patient had improvement. Therefore, it appears that the majority of immunocompetent asymptomatic patients with pulmonary cryptococcosis may undergo spontaneous remission. Six out of 31 of the asymptomatic patients did receive empirical antifungal therapy at their treating physicians' discretion. The cure rate of $100 \%$ using oral fluconazole $\left(400 \mathrm{mg} \cdot \mathrm{day}^{-1}\right.$ for $3-$ 10 months) in these six was similar to other reports from East Asia [30]. Hence, our data suggest that for most immunocompetent patients, fluconazole monotherapy is sufficient when clinically indicated, but observation alone can be justified for 
asymptomatic patients. One HIV-positive case with low CD4 count $\left(<200\right.$ cells $\left.\cdot \mathrm{mm}^{-3}\right)$ died of cryptococcal meningitis, which is an expectable outcome.

In addition, the rate of sputum culture positivity in this study was only $2.63 \%$ (two out of 76 ); all cases were C. neoformans and no C. gattii was detected. Unfortunately, no tissues were cultured at the time of surgical resection of what is thought to be malignancies. This reinforces the recognition that there is often insufficient clinical awareness of the broader differential diagnosis of abnormal radiological findings found in asymptomatic patients without overt risk factors for opportunistic pulmonary infections. Therefore, once tissues are obtained, culture should be done at the same time in order to exclude infectious diseases accurately.

\section{Conclusions}

In our retrospective series and review of literature, in the last decade, pulmonary cryptococcosis in immunocompetent persons is becoming more commonly identified. The risk factors might be related to fungus-contaminated environmental exposure. False-positive ${ }^{18}$ FDG-PET examinations often lead to initial clinical misdiagnosis of cancer. A careful understanding of the diagnostic options available can lead to fewer lung resections of nonmalignant diseases as the first invasive procedure. Lung lesions occur mostly in the outer lung fields, varying in shape. Nodular lung masses were relatively common in people with normal immune functions. For patients with tissue-confirmed pulmonary cryptococcosis and normal immune function, some exhibited spontaneous remission and the majority responded well to fluconazole monotherapy; further prospective studies should be directed towards understanding who may be carefully observed without pharmacological interventions.

\section{SUPPORT STATEMENT}

The study was funded by grants from the National Science Foundation of China (grants 30800405, 30971323 and 81170011), the Science and Technology Commission of Shanghai Municipality (grants 11430702100, 114119b2200 and 09411951500) and the International Science and Technology Cooperation Program of China (grant 2011DFB30010).

\section{STATEMENT OF INTEREST}

None declared.

\section{ACKNOWLEDGEMENTS}

The authors thank D. Li (Division of Medical and Regulatory Affairs, Wako Diagnostics Inc., Mountain View, CA, USA) and X. Wang (Dept of Preventive Medicine, Inner Mongolia Medical School, Inner Mongolia, China) for their helpful discussions.

\section{REFERENCES}

1 Perfect JR, Dismukes WE, Dromer F, et al. Clinical practice guidelines for the management of cryptococcal disease: 2010 update by the infectious diseases society of america. Clin Infect Dis 2010; 50: 291-322.

2 Galanis E, Macdougall L. Epidemiology of Cryptococcus gattii, British Columbia, Canada, 1999-2007. Emerg Infect Dis 2010; 16 251-257.

3 Hajjeh RA, Conn LA, Stephens DS, et al. Cryptococcosis: populationbased multistate active surveillance and risk factors in human immunodeficiency virus-infected persons. Cryptococcal Active Surveillance Group. J Infect Dis 1999; 179: 449-454.
4 Chang WC, Tzao C, Hsu HH, et al. Pulmonary cryptococcosis: comparison of clinical and radiographic characteristics in immunocompetent and immunocompromised patients. Chest 2006; 129: 333-340.

5 Lindell RM, Hartman TE, Nadrous HF, et al. Pulmonary cryptococcosis: CT findings in immunocompetent patients. Radiology 2005; 236: 326-331.

6 Vilchez RA, Irish W, Lacomis J, et al. The clinical epidemiology of pulmonary cryptococcosis in non-AIDS patients at a tertiary care medical center. Medicine 2001; 80: 308-312.

7 Dixit A, Carroll SF, Qureshi ST. Cryptococcus gattii: an emerging cause of fungal disease in north america. Interdiscip Perspect Infect Dis 2009; 2009: 840452.

8 Kwon-Chung KJ, Boekhout T, Fell JW, et al. Proposal to conserve the name Cryptococcus gattii against $C$. hondurianus and $C$. bacillisporus. (Basidiomycota, Hymenomycetes, Tremellomycetidae). Taxon 2002; 51: 804-806.

9 Feng X, Yao Z, Ling B, et al. Analysis of the varieties, genotypes and mating types of 110 clinical cryptococcal isolates from China. Chin J Microbiol Immunol 2008; 28: 193-197.

10 Barreto de Oliveira MT, Boekhout T, Theelen B, et al. Cryptococcus neoformans shows a remarkable genotypic diversity in Brazil J Clin Microbiol 2004; 42: 1356-1359.

11 Meyer W, Aanensen DM, Boekhout T, et al. Consensus multilocus sequence typing scheme for Cryptococcus neoformans and Cryptococcus gattii. Med Mycol 2009; 47: 561-570.

12 Zhang H. Meta analysis of pulmonary cryptococcosis record in china mainland. PhD thesis, Fudan University, 2009.

13 Zhang $\mathrm{P}$, Gao X, Xie H, et al. The characteristics of $\mathrm{CT}$ imaging and diagnosis of pulmonary cryptococcosis in 42 cases with nonacquired immune deficiency syndrome. Chin J Intern Med 2009; 48 : 362-366.

14 Zeng Y, Wu X, Yi X, et al. Clinicopathologic and ultrastructural study of non-HIV-related primary pulmonary cryptococcosis in China: report of 43 cases. Ultrastruct Pathol 2011; 35: 19-25.

15 Zhu Y, Zhang J. Case report: a case of pulmonary cryptococcosis mimic lung cancer. Shanghai Med J 1981; 4: 56.

16 Yildiran ST, Saracli MA, Gönlüm A, et al. Isolation of Cryptococcus neoformans var. neoformans from pigeon droppings collected throughout Turkey. Med Mycol 1998; 36: 391-394.

17 Ruiz A, Vélez D, Fromtling RA. Isolation of saprophytic Cryptococcus neoformans from Puerto Rico: distribution and variety. Mycopathology 1989; 106: 167-170.

18 Barrs VR, Martin P, Nicoll RG, et al. Pulmonary cryptococcosis and Capillaria aerophila infection in an FIV-positive cat. Aust Vet J 2000; 78: $154-158$

$19 \mathrm{Li} \mathrm{L}$, Wang J, Zhang Q. Isolation and identification of Cryptococcus neoformans from pigeon dropping. J Clin Dermatol 2000; 29: 4-6.

20 Colom MF, Hagen F, Gonzalez A, et al. Ceratonia siliqua (carob) trees as natural habitat and source of infection by Cryptococcus gattii in the Mediterranean environment. Med Mycol 2012; 50: 67-73.

21 Passoni LF, Wanke B, Nishikawa MM, et al. Cryptococcus neoformans isolated from human dwellings in Rio de Janeiro, Brazil: an analysis of the domestic environment of AIDS patients with and without cryptococcosis. Med Mycol 1998; 36: 305-311.

22 Garrett MH, Rayment PR, Hooper MA, et al. Indoor airborne fungal spores, house dampness and associations with environmental factors and respiratory health in children. Clin Exp Allergy 1998; 28: 459-467.

23 Wilson SC, Palmatier RN, Andriychuk LA, et al. Mold contamination and air handling units. J Occup Environ Hyg 2007; 4: 483-491.

24 Dharmage S, Bailey M, Raven J, et al. Prevalence and residential determinants of fungi within homes in Melbourne, Australia. Clin Exp Allergy 1999; 29: 1481-1489. 
25 Singh N, Alexander BD, Lortholary O, et al. Pulmonary cryptococcosis in solid organ transplant recipients: clinical relevance of serum cryptococcal antigen. Clin Infect Dis 2008; 46: e12-e18.

26 Chen CJ, Lee BF, Yao WJ, et al. Dual-phase ${ }^{18}$ F-FDG PET in the diagnosis of pulmonary nodules with an initial standard uptake value less than 2.5. Am J Roentgenol 2008; 191: 475-479.

27 Chang JM, Lee HJ, Goo JM, et al. False positive and false negative FDG-PET scans in various thoracic diseases. Korean J Radiol 2006; 7 : $57-69$.
28 Tian J, Yang X, Yu L, et al. A multicenter clinical trial on the diagnostic value of dual-tracer PET/CT in pulmonary lesions using $3^{\prime}$-deoxy- $3^{\prime}-{ }^{18}$ F-fluorothymidine and ${ }^{18}$ F-FDG. J Nucl Med 2008; 49: 186-194.

29 Saag MS, Graybill RJ, Larsen RA, et al. Practice guidelines for the management of cryptococcal disease. Infectious Diseases Society of America. Clin Infect Dis 2000; 30: 710-718.

30 Choi KH, Park SJ, Min KH, et al. Treatment of asymptomatic pulmonary cryptococcosis in immunocompetent hosts with oral fluconazole. Scand J Infect Dis 2011; 43: 380-385. 